\title{
Measurement quality estimation of proton-precession magnetometers
}

\author{
A. Y. Denisov ${ }^{1}$, O. V. Denisova ${ }^{1}$, V. A. Sapunov ${ }^{1}$, and S. Y. Khomutov ${ }^{2}$ \\ ${ }^{1}$ Quantum Magnetometry Laboratory of USTU, Mira st., 19, Ekaterinburg, 620002, Russia \\ ${ }^{2}$ Geophysical Observatory “Klyuchi”, Geophysical Survey SB RAS, Koptyug av., 3, Novosibirsk, 630090, Russia \\ (Received April 29, 2005; Revised January 20, 2006; Accepted January 21, 2006; Online published June 2, 2006)
}

\begin{abstract}
A method for calculating quality estimation of the magnetic field value measured by proton-precession magnetometers from data of single measurement is presented. This method is appropriate for frequency measurement algorithms which process time-series of signal's zero crossing. Expressions for measurement error estimation are obtained taking into account signal decay and correlated noise. Presented formulas allow a result reliability to be estimated immediately in single measurement and give an opportunity for complex control of a device performance. Correlation and relaxation corrections are given. It was experimentally tested that the standard deviation of measurements is in a good qualitative agreement with presented error estimation.
\end{abstract}

Key words: Proton Overhauser Magnetometer, magnetic observatory instrumentation, measurement quality.

\section{Introduction}

One of the most commonly used high-precision methods for geomagnetic field measurement is based on the phenomena of nuclear magnetic resonance (Primdahl, 1998). Nuclear precession frequency is directly proportional to the magnitude of a measured field by means of gyromagnetic ratio, thus providing the measurements precision.

Due to high sensitivity, the geomagnetic equipment accuracy strongly depends on service conditions, such as intrinsic noise, external disturbance, the presence of instability and measured field gradient. That is why it is necessary to estimate quality of the obtained results continuously. Many modern magnetometers have such built-in functions, a numerical system of estimation or time-averaged signal/noise for example (Freedman and Roualt, 1989). The essential disadvantage of these parameters is their indirect connection with the real error.

The aim of the present paper is to construct a natural error estimation at the given confidence coefficient of acquired data taking into account specific requirements. In particular the assessment must be derived from results of single measurement and an algorithm must satisfy the requirements for processing-time, i.e. it must be sufficiently simple.

\section{The Magnetic Field Calculation}

The algorithms used in modern proton-precession magnetometers for the geomagnetic total field transform frequency, $\omega$, of precession signal to field modulus $B$ (frequency-field conversion) (Denisov et al., 1999) by processing time-series of signal's zero crossing moments. In general, a measured period is a function of time, $t_{i}$, at zero crossing:

$$
T=F\left(t_{0}, t_{1}, \ldots, t_{N}\right),
$$

Copyright (c) The Society of Geomagnetism and Earth, Planetary and Space Sciences (SGEPSS); The Seismological Society of Japan; The Volcanological Society of Japan; The Geodetic Society of Japan; The Japanese Society for Planetary Sciences; TERRAPUB. where $N$ is a number of recorded zero crossing over measurement. In the presence of noise, $t_{i}$ differs from proper value $t_{i}^{0}$. For quasi-harmonic signals, their difference is $\Delta t_{i} \cong \pm U_{i} /\left(\omega S_{i}\right)$, where $U_{i}$ and $S_{i}$ are the noise and signal amplitudes at the output of the precession recording sensor at moment $t_{i}$, respectively. The sign choice depends on a direction of signal $i$-th front. The cross correlation of fluctuations is:

$$
\left\langle\Delta t_{i} \Delta t_{j}\right\rangle \cong(-1)^{n(i+j)} \omega^{-2} \frac{\sigma^{2}}{S_{i} S_{j}} \rho\left(\left|t_{i}^{0}-t_{j}^{0}\right|\right),
$$

where $n$ is a number of precession half-period between the two neighbouring crossing, $\rho(t)=\exp \left(-t / \tau_{C}\right)$ expresses the normed correlation function for receiving circuit tuned to the signal frequency $\omega, \tau_{C}$ is correlation time of a magnetometer's receiving circuit noise, and $\sigma^{2}$ is output noise dispersion. Thus the standard deviation of the calculated geomagnetic field value for non-damped signal and uncorrelated Gaussian noise is

$$
\sigma_{B}^{0}=\frac{B}{2 \pi} \frac{\sigma}{S_{0}} \sqrt{\sum_{j=0}^{N}\left[\frac{\partial F}{\partial t_{j}}\right]^{2}}
$$

Standard deviation expressed by Eq. (3) is an unbiased parameter of measurement quality at the given conditions. The calculated field value is assumed to follow a normal distribution. In this case, above formula for $\sigma_{B}^{0}$ allows one to estimate the confidence interval under the given confidence coefficient, i.e. to assess the data reliability. Further we will obtain error estimation at the confidence coefficient 0.68 , which is equivalent to the standard deviation.

\section{Quality Measurement Criterion (QMC)}

Assuming the noise characteristics to be time-invariant, one can estimate $\sigma_{B}^{0}$ immediately from the single measurement. For this purpose the average signal to noise ratio over 
observed precession time is needed. The problem of signal to noise estimation calls for a separate consideration. We offer to use a statistical analysis of finite difference $\Delta \tau_{i}=\left(t_{i+[2 N / 3]+k}-t_{i+k}\right)-\left(t_{i+[2 N / 3]}-t_{i}\right)$, where [...] means the integer division. The interval between time samples is $n k / 2$ periods. This value involves several correlation times of sensor receiving circuit $\tau_{C}$. In this case the calculated estimation is insensitive to slowly changing drift of an external field and gives consideration to high-frequency noise disturbances, namely equipment and external noise. The signal to noise ratio is expressed in terms of variance $\left\langle\Delta \tau^{2}\right\rangle$ :

$$
\sigma / S_{0}=\pi T^{-1} \sqrt{\left\langle\Delta \tau^{2}\right\rangle}
$$

where, for acceleration of computational process, $\left\langle\Delta \tau^{2}\right\rangle^{1 / 2}$ may be expressed in terms of sample absolute deviation $\overline{|\Delta \tau|}$

$$
\begin{aligned}
\sqrt{\left\langle\Delta \tau^{2}\right\rangle} & \cong \sqrt{\frac{\pi}{2}} \overline{|\Delta \tau|} \\
& =\frac{\sqrt{\pi / 2}}{N-[2 N / 3]-k} \sum_{i=0}^{N-[2 N / 3]-k}\left|\Delta \tau_{i}\right| .
\end{aligned}
$$

This formula is valid, provided $T_{m} \gg \tau_{C}$, where $T_{m}=$ $t_{N}-t_{0}$ is total measurement time. Thus, the formula for calculating a quality measurement criterion (QMC) is in a form:

$$
\sigma_{B}^{Q M C}=\sqrt{\frac{\pi}{2} \frac{|\Delta \tau|}{2 T}} \sqrt{\sum_{j=0}^{N}\left[\frac{\partial F}{\partial t_{j}}\right]^{2}} .
$$

As noted above, there are many algorithms processing timeseries of signal's zero crossing moments. The least squares method (Farrell and Grosch, 1965) is taken as an example as one of the most precise (Hancke, 1990):

$$
\begin{aligned}
& F\left(t_{0}, t_{1}, \ldots, t_{N}\right) \\
& \quad=\frac{12}{n N(N+1)(N+2)} \sum_{i=0}^{N}(2 i-N) \cdot t_{i} .
\end{aligned}
$$

If we substitute (7) into (6), a QMC for this method is obtained as:

$$
\sigma_{B}^{Q M C}=\frac{\sqrt{6 \pi}}{n} \frac{\overline{|\Delta \tau|}}{T} \frac{B}{\sqrt{N(N+1)(N+2)}} .
$$

\section{Relaxation Correction}

As already mentioned above, the expression (8) obtained in the previous section is appropriate for undamped signal. In order to consider a case with a decaying signal, it is necessary to determine proton magnetic relaxation time $T_{2}$ under measurement conditions, though it requires an equipment complication. Therefore, it is interesting to estimate the correction to formula (8), taking into account the relaxation process. Relying on (7) the correct standard deviation for exponential damped signal and uncorrelated noise is obtained in the form

$$
\begin{gathered}
\sigma_{B}=\sigma_{B}^{0}\left(3 e^{x} / x\right)^{1 / 2}\left(\operatorname{sh}(x)\left(1+2 x^{-2}\right)-2 x^{-1} \operatorname{ch}(x)\right)^{1 / 2} \\
x=T_{m} / T_{2}
\end{gathered}
$$

The quality criterion expressed by Eq. (8) in this case is approximately equal to

$$
\sigma_{B}^{Q M C} \cong \sigma_{B}^{0} \frac{3}{x}\left(1-e^{x / 3}\right) \sqrt{\left(e^{4 / 3 x}+1\right) / 2}
$$

The correction factor $\xi=\sigma_{B} / \sigma_{B}^{\mathrm{QMC}}$ is found as a function of $T_{m} / T_{2}$ from comparison (9) and (10). In particular for normal operating conditions the inequality $T_{m} / T_{2} \leq 2$ is satisfied and correction factor $\xi$ is close to 1 . This result allows us to conclude that the QMC is close to real estimation of standard deviation in spite of relaxation characteristics of the precession signal.

\section{Correlation Correction}

The real fluctuating noise of zero crossing is correlated with precession signal due to limited bandwidth of the magnetometer's circuit. Taking into account such fact, the measurement criterion expressed by Eq. (8) needs the correlation correction for quantity agreement with standard deviation of the repeated measurement results. For non-damped signal and correlated noise the standard deviation is in a form

$$
\sigma_{B}=\sigma_{B}^{0} \beta_{C},
$$

where $\beta_{C}$ indicates correlation correction. Generally the correction depends on a frequency of the proton signal and electrical parameters of the receiving circuit. However for the tuned to resonance receiving circuit $\beta_{C}$ is the function only of $Q$ and taking into account the relation $\tau_{C}=2 \cdot Q / \omega$ :

$$
\begin{aligned}
& \beta_{C}^{2}=\sum_{i=-\infty}^{+\infty}(-1)^{i n} \rho\left(\left|t_{i}^{0}\right|\right) \\
& =\frac{1-e^{-2 \pi / Q}+2 e^{-\pi / Q} \sin \left(2 \pi \sqrt{1-1 /\left(4 Q^{2}\right)}\right) \sqrt{4 Q^{2}-1}}{1+e^{-2 \pi / Q}-2 e^{-\pi / Q} \cos \left(2 \pi \sqrt{1-1 /\left(4 Q^{2}\right)}\right)} .
\end{aligned}
$$

As mentioned above, QMC (8) is calculated by the long time samples spaced several correlation times apart, thus the correlation correction to QMC is equal to the $\left(\beta_{C}\right.$ expressed by Eq. (12), i.e. $\sigma_{B}=\sigma_{B}^{\mathrm{QMC}} \cdot \beta_{C}$. In Fig. 1 the correlation correction is plotted as a function of the circuit quality factor $Q$.

One can see that for high- $Q$ sensors the QMC may have the error of a few times. Generally the quality $Q$ is not fixed parameter depending on temperature, frequency $\omega$ and so on. An automatic control of the quality during a

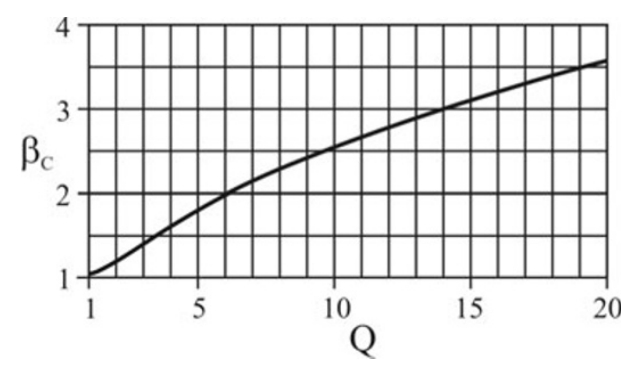

Fig. 1. The correlation correction factor $\beta_{C}$, as a function of circuit quality $Q$. 


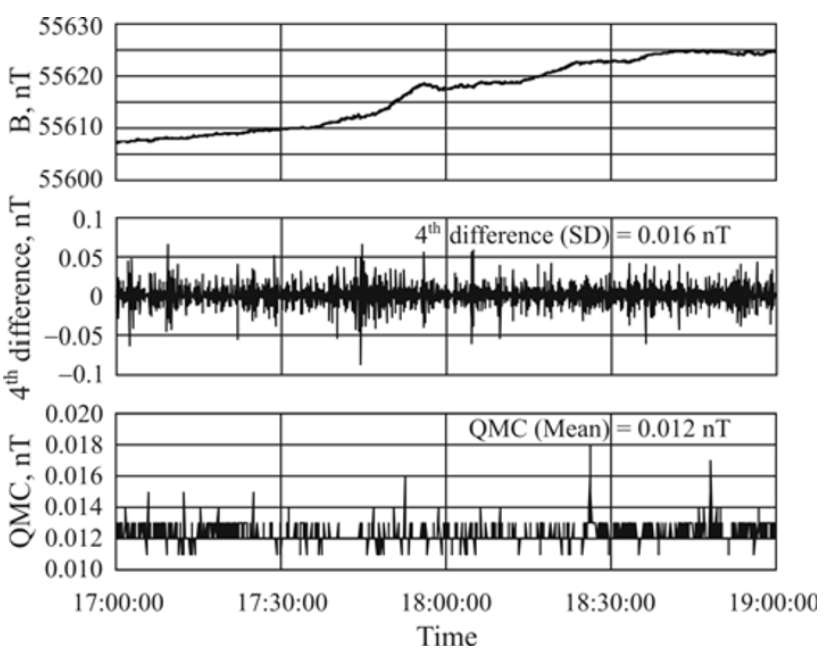

Fig. 2. Field geomagnetic variations, $B$, normalised 4th difference and QMC, experimentally recorded at the Arty Observatory, 08.09.2000. One POS sensor: $\mathrm{SD}=0.016, \mathrm{QMC}($ Mean $)=0.012$.

measurement is quite difficult and it demands complication from the magnetometer's apparatus part, but at known $Q$ the QMC can be improved according to the correction (12).

\section{Experimental Verification}

The above-presented results were experimentally tested using the POS-1 magnetometer (Processor Overhauser Sensor) and the POS-2 gradiometer serially produced by Quantum Magnetometry Laboratory since 1998. The QMC expressed by Eq. (8) is build-in function in these devices. A number of experiments were carried out to compare QMC and the real standard deviation of natural magnetic field measurements at the Arty observatory (Russia). During the first experiment the values of QMC and the magnetic variations were recorded by the POS-1 magnetometer and the time trend of the magnetic field was eliminated by means of normalized forth difference (Fig. 2).

Next investigations were made using POS-2 gradiometer. In this case, the field variations were excluded by calculating the difference between readings of two sensors (Fig. 3).

As can be seen from the experiment the real standard deviation of field measurements estimated from the processed data is in a good qualitative agreement with the mean value of the QMC. Taking into account that the POS's quality $\mathrm{Q}$ during the observations was in the range between 3 and 5 , some quantitative disagreement may be explained by the correlation correction (Fig. 1).

The practical use of QMC for observatories was revealed during many years at the observatory Klyuchi (Novosibirsk, Russia). The value of QMC was registered together with the total field by the POS-1 magnetometer and was controlled visually by magnetologist during processing. The normal QMC for the observatory is 10-12 pT but the periods of significant increasing of QMC at seeming non-disturbed total field were detected (Fig. 4). The periodical character of the QMC raises and their appearance at the peak hours, at the beginning or end of an hour, more frequently in winter and so on, allow us to connect such abnormal QMC behaviors with some technogenouse disturbances. The sensor failure

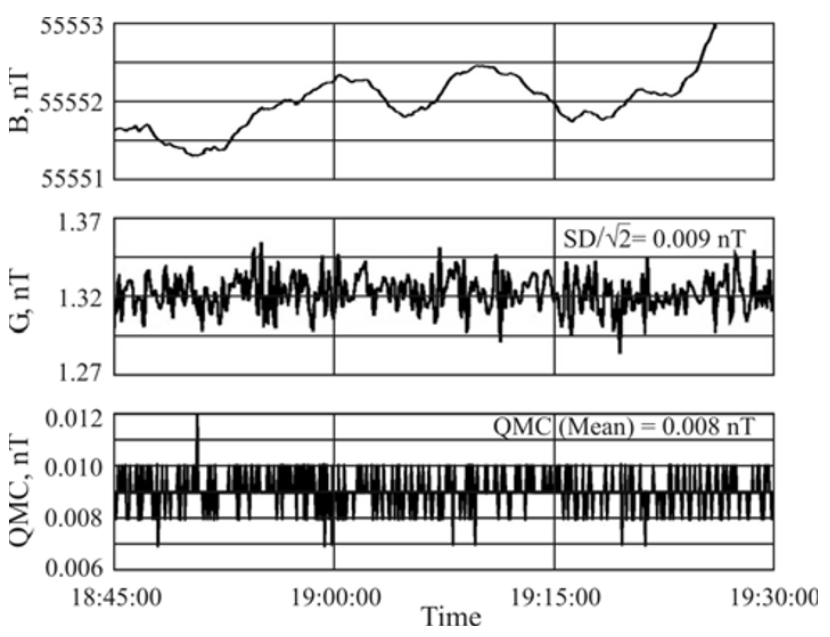

Fig. 3. Field geomagnetic variations, $B$, experimentally recorded and field difference, $G$, between sensors spaced $1.8 \mathrm{~m}$ apart at the Arty Observatory, 07.01.1999. One POS sensor: $\mathrm{SD}=0.009$, QMC $($ Mean $)=0.008$.
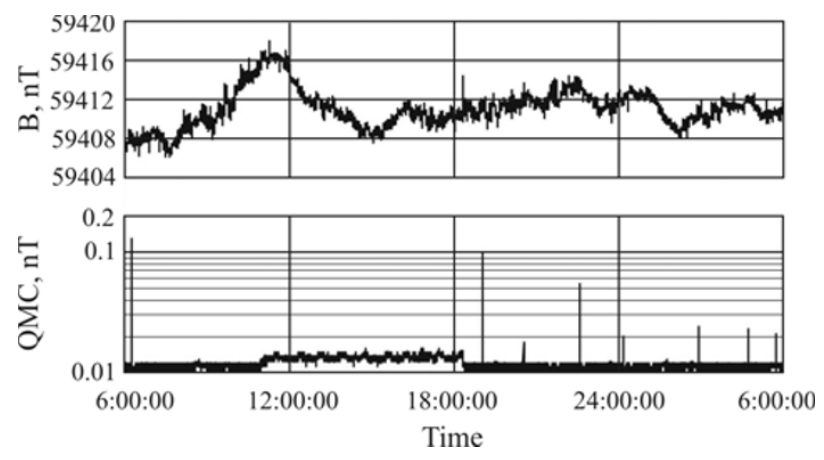

Fig. 4. The sensitivity of the QMC to technogenouse noise. Total field, $B$, and QMC, experimentally recorded at the Klyuchi Observatory, 20.02.2004.
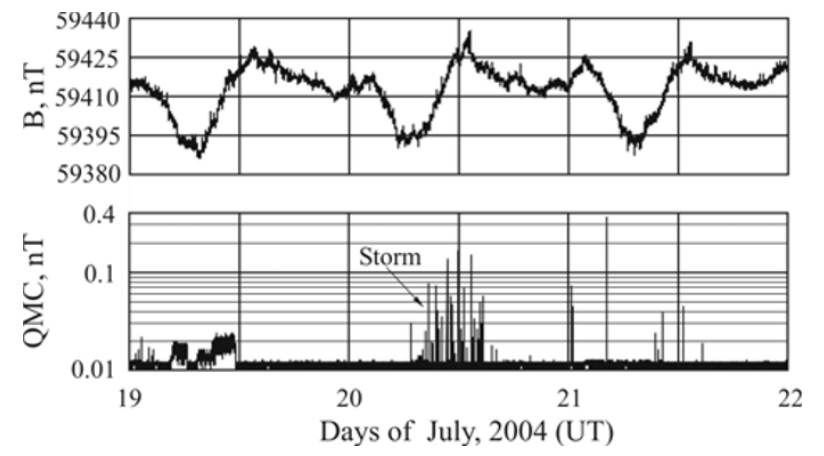

Fig. 5. The sensitivity of the QMC to thunderstorm. Total field, $B$, and QMC, experimentally recorded at the Klyuchi Observatory, 19-21.07.2004.

was excluded by the simultaneous measurements with two POS-1 and increasing QMC was at the both records. Unfortunately the source of this noise at the observatory Klyuchi is not fully identified.

QMC is sensitive not only to industrial noise but also to some meteorological effects such as thunderstorm. The thunderstorm passing close to the observatory (up to a few kilometers) in the evening of July, 20 (about 13 UT) was 
found to have disturbed QMC significantly (Fig. 5). On the contrary the total field was insensitive to the meteorological effect.

Additional application of QMC in observatory is to select a preferable position of the sensor and to test the pillars. For example at the Klyuchi observatory, four pillars were examined by the POS-1 with respect to noise level estimated by QMC and one of them with the smallest value of QMC was chosen as the permanent place of the sensor.

\section{Conclusions}

As shown in the previous section, the expressions for the quality measurement criterion QMC presented in this paper provide us a reliability to be estimated immediately in single measurement excepting long-period variations in the geomagnetic field. The QMC expressed by Eq. (6) gives an opportunity for complex control of a device performance when the admissibility of signal/noise and the signal duration decrease. The latter is possible, because the QMC is sensitive to shortening relaxation time caused by high gradient of measured magnetic field. It should be emphasized that build-in function of QMC is in a good qualitative agreement with the real measurement conditions. Some quantitative difference between QMC and standard deviation of each measurement can be ascribed to neglecting correlation correction.

\section{References}

Denisov, A. Y., V. A. Sapunov, and O. V. Dikusar, Calculation of the error in the measurements of a digital-processor nuclear-precession magnetometer, Geomagnetism and Aeronomy, 39(6), 737, 1999.

Farrell, E. and C. Grosch, Determination of period from times of zeros, Proceeding of the IEEE, 12, 2162-2163, 1965.

Freedman, R. and G. Roualt, Remaining-oil determination using nuclear magnetism logging, SPE Formation Evaluation, June, 121-130, 1989.

Hancke, G. P., The optimal frequency estimation of noise sinusoidal signal, IEE Transactions on Instrumentation and Measurement, 39(6), 843846, 1990.

Primdahl, F., Scalar magnetometers for space applications, Measurement Techniques in Space Plasmas: Field, Geophysical Monograph, 103, pp. 85-99, 1998.

A. Y. Denisov, O. V. Denisova, V. A. Sapunov (e-mail: sva@dpt.ustu. ru), and S. Y. Khomutov 